\title{
Lower weight-for-age $z$ score adversely affects hospital length of stay after the bidirectional Glenn procedure in 100 infants with a single ventricle
}

\author{
Jeffrey B. Anderson, MD, MPH, ${ }^{a}$ Robert H. Beekman III, MD, ${ }^{a}$ William L. Border, MBChB, MPH, ${ }^{a}$ \\ Heidi J. Kalkwarf, PhD, ${ }^{\mathrm{d}}$ Philip R. Khoury, MS, ${ }^{\mathrm{a}}$ Karen Uzark, PhD, ${ }^{\mathrm{a}}$ Pirooz Eghtesady, MD, PhD, ${ }^{\mathrm{b}}$ and \\ Bradley S. Marino, MD, MPP, MSCE ${ }^{\text {a,c }}$
}

\begin{abstract}
Objective: Poor growth has been described in infants with a single ventricle; however, little is known regarding its effect on surgical outcomes. We sought to assess the effect of nutritional status at the time of the bidirectional Glenn procedure on short-term outcomes.

Methods: We performed a retrospective case series of children who underwent the bidirectional Glenn procedure at our institution between January 2001 and December 2007. Anthropometric measurements were recorded at the time of neonatal admission and the bidirectional Glenn procedure. Data from preoperative echocardiograms and cardiac catheterization were recorded. The primary outcome variable was length of hospital stay.

Results: Data on 100 infants were included for analysis. Age at the time of the bidirectional Glenn procedure was 5.1 months (range, 2.4-10 months). The median weight-for-age $z$ score at birth was -0.4 (range, -2.6 to 3.2 ), and by the time of the bidirectional Glenn procedure, it had decreased to -1.3 (range, -3.9 to 0.6 ). In multivariable modeling longer postoperative hospital stays were predicted by lower weight-for-age $z$ score $(P=.02)$, younger age $(P<.001)$, being fed through a gastrostomy tube $(P=.01)$, and undergoing concomitant aortic arch reconstruction $(P<.001)$ at the time of the bidirectional Glenn procedure.
\end{abstract}

Conclusions: There is suboptimal weight gain between neonatal discharge and the bidirectional Glenn procedure. A lower weight-for-age $z$ score and younger age at the time of the bidirectional Glenn procedure affects length of hospital stay independent of hemodynamic or echocardiographic variables.

Supplemental material is available online.

Failure to thrive is common in infants with congenital heart disease (CHD). Causes that have been proposed for this phenomenon include inadequate caloric intake, increased energy expenditure, intestinal malabsorption, and decreased splanchnic blood flow. ${ }^{1,2}$

Growth and nutrition studies in the past have linked failure to thrive to CHD. Cameron and colleagues ${ }^{3}$ reported that malnutrition was present in $70 \%$ of children with CHD and cyanosis, pulmonary overcirculation, or both. Strangway and associates ${ }^{4}$ also showed a link between cyanosis and

\footnotetext{
From the Divisions of Cardiology, ${ }^{\mathrm{a}}$ Cardiothoracic Surgery, ${ }^{\mathrm{b}}$ Critical Care Medicine, and General and Community Pediatrics, ${ }^{\mathrm{d}}$ Cincinnati Children's Hospital Medical Center, Cincinnati, Ohio.

Received for publication Sept 29, 2008; revisions received Dec 15, 2008; accepted for publication Feb 16, 2009; available ahead of print May 25, 2009.

Address for reprints: Jeffrey B. Anderson, MD, MPH, Division of Cardiology, Cincinnati Children's Hospital Medical Center, 3333 Burnet Ave, ML2003, Cincinnati, OH 45259 (E-mail: jeffrey.anderson@cchmc.org).

J Thorac Cardiovasc Surg 2009;138:397-404

$0022-5223 / \$ 36.00$

Copyright (c) 2009 by The American Association for Thoracic Surgery

doi:10.1016/j.jtcvs.2009.02.033
}

poor growth. Increased energy expenditure has been implicated in infants with cyanotic CHD, including tetralogy of Fallot and total anomalous pulmonary venous connection. ${ }^{5}$ Failure to thrive is especially prevalent among infants with a single ventricle. ${ }^{6}$ These patients, who typically undergo a series of palliative surgical procedures, ${ }^{7-9}$ have difficulty achieving adequate nutritional and weight-gain goals between surgical stages. ${ }^{6}$

Poor nutrition, as defined by low fat-free mass, low body mass index, or low serum albumin or prealbumin levels, is associated with longer hospital stays and increased rate of readmission in adult patients. ${ }^{10,11}$ Increased morbidity, including increased rate of infection, has also been shown in malnourished hospitalized adults. ${ }^{12}$ Similarly, poor nutrition, as defined by low weight for age, has been linked to increased mortality in children admitted to the hospital with serious infections. ${ }^{13}$ Few studies have looked at the relationship between nutritional status and surgical outcomes in children undergoing cardiac surgery. Leite and coworkers ${ }^{14,15}$ found increased rates of mortality and postsurgical infection in a group of patients with diverse types of CHD, with poor nutrition defined as low serum albumin levels or low weight for age.

The cause of poor growth and malnutrition is poorly understood in the single-ventricle population. It is known 


\section{Abbreviations and Acronyms \\ BDG = bidirectional Glenn \\ CHD $=$ congenital heart disease \\ HLHS = hypoplastic left heart syndrome \\ WAZ $=$ weight-for-age $z$ score}

that these infants have a lower than desired total caloric intake and that their growth improves with the introduction of high caloric enteral feeds. ${ }^{6}$ It has also been shown that infants with a single ventricle have more oral-motor feeding difficulties and are slower to reach goal feeds. ${ }^{16}$ Finally, energy expenditure is increased during the initial hours after first-stage palliation in infants with a single ventricle ${ }^{17}$ but has not been evaluated thereafter.

Over the past 10 to 15 years, there have been improvements in surgical techniques, postoperative management, and perfusion strategies that have led to significantly improved survival after first-stage palliation in infants with a single ventricle. ${ }^{18}$ There are no prior studies, to our knowledge, relating nutritional status and weight gain to perioperative morbidity in patients undergoing the bidirectional Glenn (BDG) procedure or Fontan completion.

The purpose of this study was to identify specific anthropometric parameters that predict poorer short-term surgical outcomes and perioperative morbidity in infants with a single ventricle after the $\mathrm{BDG}$ procedure.

\section{MATERIALS AND METHODS \\ Study Design}

This study was a retrospective case series including all patients who underwent the BDG procedure at Cincinnati Children's Hospital Medical Center between July 2001 and December 2007. This study was approved by the Institutional Review Board at Cincinnati Children's Hospital Medical Center (06-10-30). Study data were collected from hospital medical records and Heart Institute outpatient records, as well as cardiac catheterization, echocardiographic, and cardiac surgical databases. Exclusion criteria included premature birth (<35 weeks' gestational age); chromosomal abnormalities; major congenital anomalies of the central nervous, gastrointestinal, and/or pulmonary systems; age greater than 12 months at the time of the BDG procedure; or transfer to another institution before discharge.

\section{Data Collection}

Demographics. Demographic data collected included sex, gestational age, and race.

Anthropometric measurements and nutritional management. Anthropometric measures included absolute weight and weight-for-age $z$ scores (WAZs) at initial neonatal hospital admission and average daily weight gain during neonatal hospitalization, as well as weight, length, body mass index, and $z$ scores for age at the time of the BDG procedure. Weight and length $z$ scores for age were calculated by using the Centers for Disease Control Epi-Info program (Centers for Disease Control, Atlanta Ga). The feeding route, as well as the caloric density of formula, were noted both at the time of neonatal discharge and again at the time of the BDG procedure.
Pre-BDG procedure medical and surgical management. Information regarding medical and surgical management before the BDG procedure was recorded, including initial cardiac diagnosis, previous operations and hospital courses, episodes of necrotizing enterocolitis and vocal cord paralysis, home medications, and use of supplemental oxygen before the BDG procedure.

Echocardiographic and hemodynamic data. The findings from the most recent echocardiogram performed before the $\mathrm{BDG}$ procedure were reviewed. Atrioventricular valve regurgitation and aortic or neoaortic regurgitation were considered significant if graded as moderate or greater. Residual defects, including restrictive atrial septal defect $(>2 \mathrm{~mm} \mathrm{Hg}$ gradient) and aortic arch obstruction ( $>20 \mathrm{~mm} \mathrm{Hg}$ peak corrected gradient), were noted. Decreased ventricular function was defined as moderate or greater systolic dysfunction in a right or left single ventricle or if there was a shortening fraction of less than $28 \%$ in any left ventricle.

Data from the most recent cardiac catheterization performed before the BDG procedure were reviewed. Measurements recorded included mean pulmonary artery pressure, transpulmonary gradient, pulmonary vascular resistance, ventricular end-diastolic pressure, aortic arch systolic pressure gradient, systemic arterial and mixed venous oxygen saturation, and hemoglobin level.

Operative data. Operative variables recorded included intraoperative mortality, cardiopulmonary bypass time, aortic crossclamp time, circulatory arrest time, regional low-flow cerebral perfusion time, and additional procedures performed.

Perioperative data. Variables recorded from the postoperative course included postoperative mortality; total ventilator time (time from operating room to extubation); chest tube duration; documented infections, including culture-positive bacteremia, urinary tract infections, and pneumonia, as defined by positive endotracheal tube aspirate with chest radiographic changes consistent with pneumonia; pleural effusions requiring change in diuretic regimen or insertion of a new chest tube; episodes of reintubation; total intensive care unit days; and total hospital days.

\section{Statistical Analyses}

The primary outcome variable assessed was total length of hospital stay. Secondary outcome variables included ventilator time, chest tube duration, significant infections, significant pleural effusions, and episodes of reintubation. Statistical analysis was performed with STATA 10.0 analysis software (StataCorp, College Station, Tex). All continuous variables were described and tested for central tendency to determine the normality of the data distribution. Normally distributed continuous variables are expressed as means \pm standard deviations. Nonnormally distributed data are expressed by using the median value and range. Univariate analyses of potential risk factors for adverse outcome were performed by using the Wilcoxon rank sum test for continuous variables and the Fisher's exact test for dichotomous variables. Candidate predictor variables for multivariable modeling had a $P$ value of less than .1 on bivariate analysis. Multivariable analyses were performed by using linear and logistic regression. A correlation table was used to assess for colinearity of variables.

\section{RESULTS Study Population}

A total of 120 patients underwent the BDG procedure at our institution during the study period. Twenty patients were excluded for the following reasons: prematurity of less than 35 weeks' gestation $(n=4)$; chromosomal abnormalities $(\mathrm{n}=7)$; major central nervous, gastrointestinal, or pulmonary system anomalies $(\mathrm{n}=2)$; age greater than 12 months at the time of the BDG procedure $(\mathrm{n}=4)$; and transfer to another institution before discharge after the BDG 
TABLE 1. Patient characteristics

\begin{tabular}{|c|c|c|}
\hline \multirow[t]{2}{*}{$\overline{S e x}$} & Male & $56 \%$ \\
\hline & Female & $44 \%$ \\
\hline Gestational age (wk) & $40(35-40)$ & \\
\hline Birth weight (kg) & $3.1(2.2-4.4)$ & \\
\hline \multirow[t]{3}{*}{ Race } & White & $84^{\circ}$ \\
\hline & African American & $13^{\circ}$ \\
\hline & Other & 3 \\
\hline \multirow[t]{6}{*}{ Neonatal surgery } & No neonatal surgery & $12 \%$ \\
\hline & BTS alone & $22^{\circ}$ \\
\hline & PA band alone & $7^{\circ}$ \\
\hline & Arch reconstruction & $59^{\circ}$ \\
\hline & With BT shunt & $50 \%$ \\
\hline & With Sano shunt & $9^{\circ}$ \\
\hline Age at BDG surgery (mo) & $5.1(2.4-10)$ & \\
\hline \multicolumn{2}{|l|}{ Dominant right ventricle } & \\
\hline \multicolumn{2}{|l|}{ Hypoplastic left heart syndrome } & 37 \\
\hline \multicolumn{2}{|l|}{ RV-dominant AV canal } & \\
\hline \multicolumn{2}{|l|}{ DORV/mitral atresia } & \\
\hline \multicolumn{2}{|l|}{$\mathrm{RV}$ to aorta/pulmonary atresia } & \\
\hline \multicolumn{2}{|l|}{ Dominant left ventricle } & 4 \\
\hline \multicolumn{2}{|l|}{ Double-inlet left ventricle } & 2 \\
\hline \multicolumn{2}{|l|}{ Tricuspid atresia } & \\
\hline \multicolumn{2}{|l|}{ Pulmonary atresia/IVS } & \\
\hline \multicolumn{2}{|l|}{ LV-dominant AV canal } & \\
\hline Other & & \\
\hline
\end{tabular}

$\overline{B T S \text {, Blalock-Taussig shunt; } P A \text {, pulmonary artery; } B D G \text {, bidirectional Glenn proce- }}$ dure; $R V$, right ventricle; $A V$, atrioventricular; $D O R V$, double-outlet right ventricle; $I V S$, intact ventricular septum; $L V$, left ventricle.

procedure $(\mathrm{n}=3)$. Therefore there were 100 patients who met inclusion criteria. Patient characteristics are shown in Table 1. All patients undergoing superior cavopulmonary anastomosis had a BDG shunt, and 7 patients received bilateral BDG shunts; there were no hemi-Fontan procedures performed.

\section{Anthropometric Measurements and Nutritional Parameters}

At neonatal admission, infants in our study had a median weight of $3.1 \mathrm{~kg}$ (range, $2.2-4.4 \mathrm{~kg}$ ) and a median WAZ of -0.3 (range, -2.6 to 3.2). At the time of the BDG procedure, the median weight was $5.7 \mathrm{~kg}$ (range, $3.6-8.4 \mathrm{~kg}$ ), and the median WAZ decreased to -1.3 (range, -3.9 to 0.6 ), as demonstrated in Figure 1. The median difference in WAZs between birth and the BDG procedure was -1.2 (range, -4.7 to 1.1). Similarly, the median $z$ score for length at the time of the BDG procedure was -1.5 (range, -4 to 1.3 ).

During the first 5 months of life, the average daily weight gain for a healthy infant, according to the Centers for Disease Control and Prevention databases, is 22.5 g. ${ }^{19}$ In our cohort the median daily weight gain was $16.5 \mathrm{~g} / \mathrm{d}$ (range, $8.9-30.3 \mathrm{~g} / \mathrm{d}$ ), and $89 \%$ of our patients failed to meet recommended Centers for Disease Control and Prevention standards (see Figure E1).

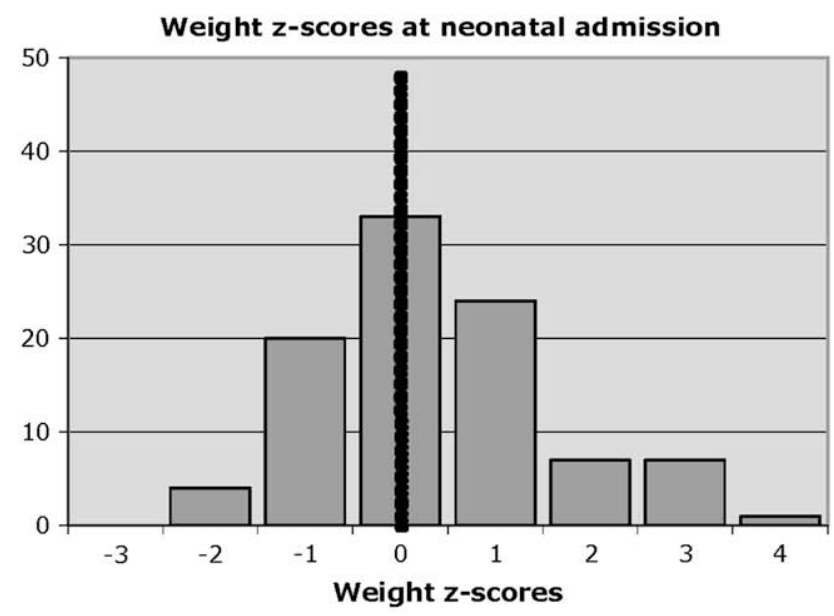

Weight z-scores at BDG

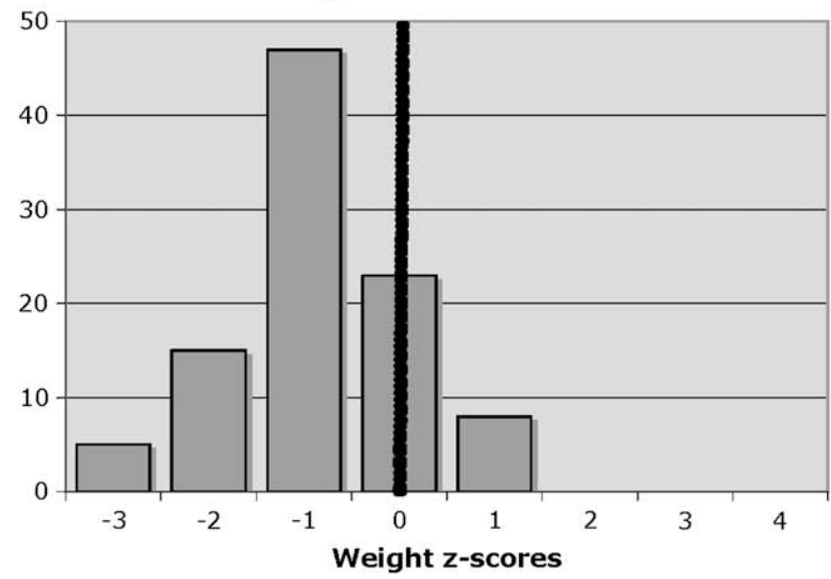

FIGURE 1. Weight-for-age $z$ scores at birth and at the time of the bidirectional Glenn $(B D G)$ procedure are shown. There was a significant trend toward the lower end of the growth curve over time.

\section{Pre-BDG Medical and Surgical Management}

There were 95 patients who required neonatal hospitalization for a median hospital length of stay of 20 days (range, 3100 days). During the neonatal hospitalization, there were 5 patients with necrotizing enterocolitis and 17 patients with documented vocal cord paralysis. It is policy at our institution to perform fibroscopic airway evaluation of all infants who have undergone the Norwood procedure before their discharge from the hospital. At the time of the BDG procedure, $97 \%$ of patients were receiving diuretics, $56 \%$ were receiving an angiotensin-converting enzyme inhibitor, and $38 \%$ were receiving digoxin. Only $10 \%$ of the patients were receiving supplemental oxygen.

A comparison of feeding plans at neonatal discharge and just before the BDG procedure is shown in Figure E2. Information was available on feeding plans for $94 \%$ of the cohort at neonatal discharge and $94 \%$ of the cohort at the time of the BDG procedure. The number of patients fed orally exclusively increased from $25 \%$ to $69 \%$, whereas the number of 
patients supported with either nasogastric or gastrostomy tube feedings decreased from $75 \%$ to $35 \%$. A majority of patients were fed formula, with only $24 \%$ receiving breast milk at neonatal discharge and $9 \%$ at the time of the BDG procedure. Formula and breast milk were fortified to an average of $25.2 \mathrm{kcal} / \mathrm{oz}$ at neonatal discharge and $24.9 \mathrm{kcal} / \mathrm{oz}$ at the time of the BDG procedure. Feeding plans were not standardized and were left to the discretion of individual cardiologists and pediatricians.

There were 8 patients who had placement of gastrostomy tubes, 4 during their neonatal hospitalization and 4 during the interstage period. All of these patients had hypoplastic left heart syndrome (HLHS) or a variant and previously underwent a Norwood procedure with aortic arch reconstruction, leading to longer neonatal length of stay (36 vs 20 days, $P=.02)$. In addition, this group required a higher caloric formula (27 vs $24 \mathrm{kcal} / \mathrm{oz}, P=.007$ ) in the interstage period.

Those patients with HLHS $(\mathrm{n}=37)$ had WAZs similar to those of the remainder of the cohort at neonatal admission $(P=.6)$ and at the time of the BDG procedure $(P=.6)$, but there was a trend in this group toward younger age at the time of the BDG procedure (4.8 vs 5.1 months, $P=.08$ ). This group had a longer neonatal hospital length of stay (24 vs 20 days, $P=.007$ ) and required higher caloric intake ( $27 \mathrm{vs} 24 \mathrm{kcal} / \mathrm{oz}, P=.007)$ in the interstage period. There was no difference in pre-BDG procedure oxygen saturation $(P=.09)$ or age at the time of the BDG procedure $(P=.25)$ in those infants who had a Norwood procedure performed with a Sano shunt compared with those with a BlalockTaussig shunt. Interestingly, despite longer neonatal hospitalizations and need for higher caloric intake, having a diagnosis of HLHS was not associated with longer hospital length of stay in multivariable modeling. There were no differences in echocardiographic or hemodynamic measurements between the patients with HLHS and the remainder of the cohort.

\section{Echocardiographic and Hemodynamic Data}

All patients had echocardiograms, and 97 underwent cardiac catheterization before surgical intervention. Echocardiographic and hemodynamic findings are shown in Table 2.

\section{Operative Data}

BDG procedures with takedown of the Blalock-Taussig shunt were performed off cardiopulmonary bypass in $16 \mathrm{pa}-$ tients. In those in whom cardiopulmonary bypass was used and no additional procedures were performed $(n=46)$, the median cardiopulmonary bypass time was 70 minutes (range, 37-146 minutes). In those who had additional procedures performed during cardiopulmonary bypass $(\mathrm{n}=38)$, the median cardiopulmonary bypass time was 109 minutes (range, 57-231 minutes). The most common additional procedures performed included pulmonary arterioplasty $(\mathrm{n}=$ $19)$, atrial septectomy $(\mathrm{n}=11)$, and aortic arch reconstruc-
TABLE 2. Preoperative echocardiographic and hemodynamic data

\begin{tabular}{lcc}
\hline & Median (range) & Total \\
\hline $\begin{array}{l}\text { Echocardiographic } \\
\text { No. of days before BDG }\end{array}$ & $24(1-176)$ & \\
$\quad$ echocardiogram performed & & $20(20 \%)$ \\
Moderate or greater atrioventricular & & $5(5 \%)$ \\
$\quad$ valve regurgitation & & $13(13 \%)$ \\
Restrictive atrial septal defect & & $4(4 \%)$ \\
$\quad$ (>2 mm Hg) & & $7(7 \%)$ \\
Aortic arch gradient (>20 mm Hg) & & $1(1 \%)$ \\
Moderate or greater aortic & & \\
$\quad$ insufficiency & & \\
Right ventricular systolic dysfunction & & \\
Left ventricular systolic dysfunction & & \\
Hemodynamic data & & \\
No. of days before BDG cardiac & $22(0-129)$ & \\
$\quad$ catheterization performed & & \\
Hemoglobin (g/dL) & & \\
Mixed venous oxygen saturation & $50 \%(26 \%-65 \%)$ & \\
Systemic arterial oxygen saturation & $76 \%(60 \%-92 \%)$ & \\
Qp/Qs & $1.17(0.24-4.3)$ & \\
Mean pulmonary artery pressure & $14(6-36)$ & \\
$\quad$ (mm Hg) & & \\
Left atrial pressure (mm Hg) & $6(1-28)$ & \\
Transpulmonary gradient (mm Hg) & $7(1-21)$ & \\
Systemic ventricle end-diastolic & $7(2-16)$ & \\
$\quad$ pressure (mm Hg) & & \\
Aortic arch gradient (>20 mm Hg) & & \\
Pulmonary vascular resistance (IWU) & $1.9(0.24-5.2)$ & \\
\hline
\end{tabular}

$B D G$, Bidirectional Glenn procedure; $Q p / Q s$, ratio of pulmonary blood flow versus systemic blood flow; $I W U$, indexed Woods units.

tion $(\mathrm{n}=8)$. Six patients had pulmonary artery band removal off cardiopulmonary bypass. Aortic crossclamps for additional intracardiac procedures were used in 11 patients for a median of 19.5 minutes (range, 8-95 minutes). Aortic arch repair was performed in 8 patients, with a median regional low-flow cerebral perfusion time of 42.5 minutes (range, 23-95 minutes). Circulatory arrest was used in 5 patients undergoing aortic arch reconstruction for a median of 19 minutes (range, 2-38 minutes). In those in whom circulatory arrest was used, the circulatory arrest time was included in the regional low-flow cerebral perfusion time.

\section{Perioperative Data}

There were $3(3 \%)$ postoperative deaths. One patient with a double-outlet right ventricle, mitral atresia, and a restrictive atrial septum acutely had hypotension, bradycardia, and lactic acidosis 7 hours postoperatively, and attempts to resuscitate were unsuccessful. Autopsy showed a left-sided myocardial ischemic event. A second patient with severe valvar pulmonary stenosis and right ventricular hypoplasia underwent balloon pulmonary valvuloplasty complicated by severe hypoxemia requiring an emergency BDG procedure. Despite the BDG procedure, the patient remained severely hypoxemic 
and was started on extracorporeal membrane oxygenation. She ultimately died from complications related to extracorporeal membrane oxygenation. Finally, a third patient with HLHS who had previously had a Norwood procedure died after a prolonged postoperative BDG course that included thrombophilia, resulting in bilateral superior vena cava clots; reoperation for aortic arch obstruction and severe tricuspid valve regurgitation; hemidiaphragm paralysis; and failed extubations, resulting in tracheostomy. Ultimately, the decision was made to withdraw support rather than pursue transplantation.

Of those who survived, the median intensive care unit length of stay was 3 days (range, 1-42 days), and the median hospital length of stay was 6 days (range, 3-56 days). The median ventilator time was 16 hours (range, 0-495 hours), and chest tube duration was 2 days (range, 1-6 days). Seven patients required reintubation, and 10 patients had significant pleural effusions. There were 9 patients with serious infections, including bacteremia $(\mathrm{n}=3)$, urinary tract infection $(\mathrm{n}=3)$, and pneumonia $(\mathrm{n}=3)$.
Table 3 demonstrates the results from univariate analyses and multivariable modeling for perioperative outcome. $\mathrm{Pa}$ tients with a lower WAZ at the time of the BDG procedure $(P=.02)$ were more likely to have prolonged hospitalizations. In addition, those who were younger at the time of the BDG procedure $(P<.001)$, those who were fed through a gastrostomy tube before the BDG procedure $(P=.01)$, and those who underwent concurrent aortic arch reconstruction $(P<.001)$ were likely to have increased hospital length of stay. Those patients with a lower WAZ $(P=.07)$ also trended toward having 1 or more of any of the postoperative complications we measured (mortality, pleural effusions, infections, or failure of discharge goals).

Patients who underwent concurrent aortic arch reconstruction $(P<.001)$ and those who had a lower absolute weight at the time of the BDG procedure $(P=.01)$ were likely to have longer ventilator times. Those who had lower average daily weight gain during their neonatal hospitalization $(P=.03)$ and those fed through a gastrostomy tube at the time of the BDG procedure $(P=.001)$ were more likely

TABLE 3. Univariate analysis and multivariable modeling for predictors of worse postoperative outcome

\begin{tabular}{|c|c|c|c|c|c|}
\hline \multicolumn{2}{|c|}{ Variables } & \multicolumn{4}{|c|}{ Analyses } \\
\hline \multirow[b]{2}{*}{ Outcome } & \multirow[b]{2}{*}{ Independent } & \multirow{2}{*}{$\begin{array}{c}\text { Univariate } \\
P \text { value }\end{array}$} & \multicolumn{3}{|c|}{ Multivariate } \\
\hline & & & $P$ value & $\beta$ coefficient $(95 \%$ CI $)$ & $R^{2}$ \\
\hline \multirow[t]{12}{*}{ Hospital length of stay (d) } & Age at BDG (mo) & $<.001$ & $<.001$ & $-1.7(-2.6$ to -0.8$)$ & 0.43 \\
\hline & Weight-for-age $z$ score at BDG & .06 & .02 & $-1.7(-3.2$ to -0.19$)$ & \\
\hline & Aortic arch reconstruction at $\mathrm{BDG}$ & .03 & $<.001$ & $20.8(14.5$ to 27.1$)$ & \\
\hline & Gastrostomy tube feedings at BDG & .002 & .01 & $6.5(1.6$ to 11.4$)$ & \\
\hline & Dominant right ventricle & .07 & NS & & \\
\hline & Diagnosis of HLHS & .06 & NS & & \\
\hline & Neonatal treatment of NEC & .03 & NS & & \\
\hline & Weight gain birth to BDG (g/d) & .1 & NS & & \\
\hline & Mean PA pressure at catheterization & NS & NS & & \\
\hline & PVR at catheterization & NS & NS & & \\
\hline & Ventricular end-diastolic pressure & NS & NS & & \\
\hline & Qp/Qs at catheterization & NS & NS & & \\
\hline \multirow[t]{9}{*}{ Ventilator time (min) } & Age at BDG (mo) & .05 & .008 & $-8.4(-14.5$ to -2.2$)$ & 0.18 \\
\hline & Weight-for-age $z$ score at BDG & NS & .12 & $-8.7(-20.1$ to 2.5$)$ & \\
\hline & Aortic arch reconstruction at BDG & .02 & $<.001$ & $87.2(45.9$ to 128.5$)$ & \\
\hline & Cardiopulmonary bypass time (min) & .03 & NS & & \\
\hline & Diagnosis of HLHS & .05 & NS & & \\
\hline & Mean PA pressure by catheterization & NS & NS & & \\
\hline & PVR at catheterization & NS & NS & & \\
\hline & Ventricular end-diastolic pressure & NS & NS & & \\
\hline & Qp/Qs at catheterization & NS & NS & & \\
\hline \multirow[t]{8}{*}{ Chest tube duration (d) } & Weight-for-age $z$ score at BDG & NS & .8 & $0.03(-0.24$ to 0.31$)$ & 0.19 \\
\hline & Neonatal admission weight gain $(\mathrm{g} / \mathrm{d})$ & .04 & .03 & $-0.9(-1.6$ to -0.18$)$ & \\
\hline & Gastrostomy tube feedings at BDG & .05 & .001 & $1.3(0.62$ to 2.5$)$ & \\
\hline & Documented vocal card paralysis & .08 & NS & & \\
\hline & Mean PA pressure by catheterization & NS & NS & & \\
\hline & PVR at catheterization & NS & NS & & \\
\hline & Ventricular end-diastolic pressure & NS & NS & & \\
\hline & Qp/Qs at catheterization & NS & NS & & \\
\hline
\end{tabular}

$B D G$, Bidirectional Glenn procedure; $N S$, not significant $H L H S$, hypoplastic left heart syndrome; $N E C$, necrotizing enterocolitis; $P A$, pulmonary artery; $P V R$, pulmonary vascular resistance; $Q p / Q s$, ratio of pulmonary blood flow versus systemic blood flow. 
to have longer chest tube duration. WAZ at the time of the BDG procedure $(P>.2)$ was not associated with longer ventilator time or chest tube duration.

No hemodynamic measurements or echocardiographic findings were associated with worse postoperative outcomes. Specifically, there was no association between pulmonary vascular resistance, mean pulmonary artery pressure, or increased ventricular end-diastolic pressure with outcome measures. Performance of the BDG procedure off cardiopulmonary bypass $(P>.2)$ was not associated with better postoperative outcomes. No anatomic diagnosis, including having a dominant left or right ventricle $(P>.2)$, having a diagnosis of HLHS $(P>.2)$, or having a bilateral superior vena cava $(P>.2)$, was associated with an increased hospital length of stay. Neonatal hospital length of stay $(P>.2)$ and episodes of necrotizing enterocolitis $(P>.2)$ or vocal cord paralysis $(P>.2)$ did not predict worse outcomes. In addition, neither preoperative medication regimen nor caloric density of formula predicted worse postoperative outcomes.

\section{DISCUSSION}

Infants in our study had a normal weight distribution at birth but grew poorly and had a lower WAZ at the time of their BDG procedures. Patients with a lower WAZ at the time of the BDG procedure had a longer hospital length of stay. These findings were independent of any echocardiographic or hemodynamic measurements obtained before surgical intervention.

Age at the time of the BDG procedure for our cohort was similar to that of other studies focusing on outcomes after superior cavopulmonary anastomosis. ${ }^{20}$ Mortality in our cohort of patients was low and similar to what has been documented in other reports. ${ }^{20}$ In those patients who survived, length of hospital stay was comparable with that seen in other superior cavopulmonary anastomosis series. ${ }^{20}$ This is the first study, to our knowledge, to assess for associations between anthropometric measurements and perioperative morbidity in infants with a single ventricle who underwent the BDG procedure. Poor growth noted in this cohort was similar to what Kelleher and colleagues ${ }^{6}$ reported in infants with HLHS. We found that a lower WAZ at the time of the BDG procedure predicted a longer hospital length of stay. Studies in adults have shown an association between poor nutrition and increased hospital length of stay. ${ }^{10-12}$ Studies of hospitalized children have shown an association between malnutrition and increased morbidity and mortality. ${ }^{13,14}$ Caulfield and associates ${ }^{13}$ showed that malnourished children hospitalized with serious bacterial infections had increased mortality. Poor nutrition in hospitalized children undergoing cardiac surgery is associated with an increase in postoperative infections, ${ }^{14}$ which might lead to longer hospital length of stay. In our series an increase in the incidence of postoperative infections was not observed. However, poor nutrition can lead to lower protein reserves, causing decreased intravascular oncotic pressure and an increased frequency and severity of pleural effusions, intestinal wall edema, and ascites. In addition, poor nutrition might also result in lower levels of micronutrients, causing poor immune function, ${ }^{21}$ which can affecting normal healing, or decreased muscle mass ${ }^{11}$ and strength, hindering the ability to recover after surgical intervention. Although the retrospective nature of this study precluded us from detailed information, other factors that might have delayed hospital discharge include increased time needed to reach goal feeds and lower respiratory reserve requiring longer courses of supplemental oxygen after extubation.

Younger age at the time of surgical intervention was associated with a longer hospital length of stay. Jaquiss and coworkers $^{22}$ assessed for predictors of perioperative morbidity in a series of patients undergoing the BDG procedure and, similar to our study, found that younger patients had longer total hospital lengths of stay. In addition, Jaquiss and coworkers $^{22}$ noted that younger patients tended to have lower systemic oxygen saturation initially after the BDG procedure and theorized that there might have been more cardiopulmonary bypass-induced lung injury in this susceptible group. The association between age and length of hospital stay is particularly important because there is a current trend toward performing the BDG procedure at a younger age. ${ }^{23}$

Infants undergoing concurrent aortic arch reconstruction also had a longer total hospital length of stay. Decreased perfusion to the lower body during surgical intervention might lead to splanchnic ischemia and postoperative reperfusion injury, manifesting as the postcoarctectomy syndrome. ${ }^{24}$ Gastrointestinal manifestations of this phenomenon might lead to poor feeding tolerance and a longer time to reach feeding goals. Finally, in patients who underwent concurrent aortic arch reconstruction, clinical decisions might have been made to delay extubation in the postoperative period longer than in those patients without arch reconstruction.

In our cohort only those with severe feeding and growth difficulties underwent gastrostomy tube placement. All patients who had gastrostomy tubes placed had difficulty with feeding and poor weight gain. The decision to place a gastrostomy tube was made by individual cardiologists and pediatricians. This group had prolonged neonatal hospital courses and received higher caloric support. Those infants who had a gastrotomy tube placed did not have improved growth after its placement compared with the remainder of the cohort. We hypothesize that this was due to their high metabolic needs and growth difficulties that resulted in moving to this feeding strategy. We found that these patients also were more likely to have prolonged hospitalizations after their BDG procedures. We speculate that this group had a higher metabolic need and might have come to their BDG procedures with less nutritional stores, leading to more difficulty with postoperative recovery.

Increased cardiopulmonary bypass time has been shown to lead to a systemic inflammatory response. ${ }^{25}$ Significant 
lung growth occurs in the first 2 years of life. ${ }^{26}$ Therefore younger infants have more immature lungs, which might be more susceptible to cardiopulmonary bypass-induced injury. ${ }^{27}$ This injury might prolong pulmonary recovery and lengthen hospital stay. Similarly, systemic inflammation might affect the gastrointestinal tract, leading to feeding intolerance and difficulty reaching nutritional discharge goals. Cardiopulmonary bypass time affected discharge goals, whereas it did not affect total hospital length of stay, suggesting a threshold effect.

Patients had a median ventilator time similar to what others have reported. ${ }^{20}$ Similar to Kloth and Baum, ${ }^{28}$ we found that younger patients were more likely to have longer ventilation times. A lower functional residual capacity in smaller children might lead to a propensity for more problems with areas of intrapulmonary shunting in the postoperative period. Cardiopulmonary bypass-induced lung injury in this group with potential lung immaturity might lead to longer ventilator duration. Infants who underwent aortic arch reconstruction also had longer periods of mechanical ventilation. Although uncommon, postoperative chylothorax might be more common after arch reconstruction. ${ }^{29}$ Finally, it might be that in patients who underwent concurrent aortic arch reconstruction clinical decisions were made to delay extubation in the postoperative period.

The median chest tube duration in our series was also similar to what others have found in this population. ${ }^{30}$ Infants who were fed by means of a gastrostomy tube and those who had poor growth during their neonatal hospitalization likely had complicated neonatal courses requiring more aggressive nutritional and medical management. We hypothesize that this group had lower nutritional reserves coming into their BDG procedures, including lower albumin levels, leading to increase fluid loss to the pleural space.

There are limitations to this investigation that must be acknowledged. Because this was a retrospective case series, during the data acquisition period, there might have been specific data points that were missing. Anthropometric measures were used as markers of nutrition. Although other studies have used similar methods, ${ }^{13}$ these are indirect measures of nutritional status; more accurate biochemical markers might better predict poor nutrition. Because primary care physicians and primary cardiologists determined feeding plans, there was variability in management. This might have introduced some bias, making it more difficult to find differences that actually exist in nutritional makeup.

\section{CONCLUSIONS}

There is suboptimal growth in infants with a single ventricle between their first- and second-stage surgical palliation. Those infants with poor growth, as manifested by a lower $\mathrm{WAZ}$ at the time of their BDG procedure, had longer hospital stays. In addition, younger infants, independent of WAZ, were also more likely to have prolonged hospitalizations. No echocardiographic or hemodynamic factors predicted longer hospital length of stay.

These findings are clinically important because as we better understand the physiology of poor growth in this population and improve feeding strategies, we might be able to improve outcomes after the BDG procedure. Additional work in this area will add to our limited understanding of the physiology of growth failure in patients with a single ventricle. By using both retrospective and prospective methods, risk factors need to be identified that predict poor interstage growth, and appropriate biochemical markers for nutritional failure need to be identified that might be used as adjuncts to anthropometric measures. Although short-term outcome after the BDG procedure is important, other outcomes, including subsequent weight gain, suitability for a later Fontan procedure, and cognitive and neurodevelopment outcome, might also be affected by growth, and these require further study. Ultimately, nutritional interventions need to be prospectively evaluated so that we can improve growth in this high-risk population.

With the understanding that this group of patients experiences significant difficulty with feeding and growth, attention needs to be given to their nutritional status. As we follow these patients between surgical stages, care needs to be given to their weight gain and nutritional goals. Improved weight gain will lead to improved outcome at the time of their second-stage palliation.

\section{References}

1. Gingell RL, Hornung MG. Growth problems associated with congenital heart disease in infancy. In: Lebenthal E, ed. Textbook of gastroenterology and nutrition in infancy. New York: Raven; 1981:853-860.

2. Hansen SR, Dorup I. Energy and nutrient intakes in congenital heart disease. Acta Paediatr. 1993;82:166-72.

3. Cameron JW, Rosenthal A, Olson AD. Malnutrition in hospitalized children with congenital heart disease. Arch Pediatr Adolesc Med. 1995;149:1098-102.

4. Strangway A, Fowler R, Cunningham K, Hamilton JR. Diet and growth in congenital heart disease. Pediatrics. 1976;57:75-86.

5. Leitch CA, Karn CA, Peppard RJ, Granger D, Liechty EA, Ensing GJ, et al. Increased energy expenditure in infants with cyanotic congenital heart disease. $J P e$ diatr. 1998;133:755-60.

6. Kelleher DK, Laussen P, Teixeira-Pinto A, Duggan C. Growth and correlates of nutritional status among infants with hypoplastic left heart syndrome (HLHS) after stage 1 Norwood procedure. Nutrition. 2006;22:237-44.

7. Norwood WI, Lang P, Hansen DD. Physiologic repair of aortic atresia-hypoplastic left heart syndrome. $N$ Engl J Med. 1983;308:23-6.

8. Glenn WW. Superior vena cava-pulmonary artery shunt. By William W.L. Glenn, 1958. Ann Thorac Surg. 1989;47:62-4.

9. Bartmus DA, Driscoll DJ, Offord KP, Humes RA, Mair DD, Schaff HV, et al. The modified Fontan operation for children less than 4 years old. J Am Coll Cardiol. 1990;15:429-35

10. Kyle UG, Genton L, Pichard C. Hospital length of stay and nutritional status. Curr Opin Clin Nutr Metab Care. 2005;8:397-402.

11. Pichard C, Kyle UG, Morabia A, Perrier A, Vermeulen B, Unger P. Nutritional assessment: lean body mass depletion at hospital admission is associated with an increased length of stay. Am J Clin Nutr. 2004;79:613-8.

12. Naber TH, Schermer T, de Bree A, Nusteling K, Eggink L, Kruimel JW, et al Prevalence of malnutrition in nonsurgical hospitalized patients and its association with disease complications. Am J Clin Nutr. 1997;66:1232-9.

13. Caulfield LE, de Onis M, Blossner M, Black RE. Undernutrition as an underlying cause of child deaths associated with diarrhea, pneumonia, malaria, and measles. Am J Clin Nutr. 2004;80:193-8. 
14. Leite HP, Fisberg M, de Carvalho WB, de Camargo Carvalho AC. Serum albumin and clinical outcome in pediatric cardiac surgery. Nutrition. 2005;21:553-8.

15. Leite HP, Fisberg M, Novo NF, Nogueira EB, Ueda IK. Nutritional assessment and surgical risk markers in children submitted to cardiac surgery. Sao Paulo Med J. 1995;113:706-14.

16. Davis D, Davis S, Cotman K, Worley S, Londrico D, Kenny D, et al. Feeding difficulties and growth delay in children with hypoplastic left heart syndrome versus d-transposition of the great arteries. Pediatr Cardiol. 2008;29: 328-33.

17. Li J, Zhang G, Herridge J, Holtby H, Humpl T, Redington A, et al. Energy expenditure and caloric and protein intake in infants following the Norwood procedure. Pediatr Crit Care Med. 2008;9:55-61.

18. Mahle WT, Spray TL, Wernovsky G, Gaynor JW, Clark BJ 3rd. Survival after reconstructive surgery for hypoplastic left heart syndrome: a 15-year experience from a single institution. Circulation. 2000;102(suppl 3):III136-41.

19. Kuczmarski RJ, Ogden CL, Grummer-Strawn LM, Flegal KM, Guo SS, Wei R, et al. CDC growth charts: United States. Adv Data. 2000;8:1-27.

20. Brown DW, Gauvreau K, Powell AJ, Lang P, Colan SD, Del Nido PJ, et al. Cardiac magnetic resonance versus routine cardiac catheterization before bidirectional Glenn anastomosis in infants with functional single ventricle: a prospective randomized trial. Circulation. 2007;116:2718-25.

21. Corman LC. Effects of specific nutrients on the immune response. Selected clinical applications. Med Clin North Am. 1985;69:759-91.

22. Jaquiss RD, Ghanayem NS, Hoffman GM, Fedderly RT, Cava JR, Mussatto KA, et al. Early cavopulmonary anastomosis in very young infants after the Norwood procedure: impact on oxygenation, resource utilization, and mortality. $J$ Thorac Cardiovasc Surg. 2004;127:982-9.

23. Jaquiss RD, Siehr SL, Ghanayem NS, Hoffman GM, Fedderly RT, Cava JR, et al. Early cavopulmonary anastomosis after Norwood procedure results in excellent Fontan outcome. Ann Thorac Surg. 2006;82:1260-6.

24. Malagon I, Onkenhout W, Klok G, van der Poel PF, Bovill JG, Hazekamp MG. Gut permeability in paediatric cardiac surgery. $\mathrm{Br}$ J Anaesth. 2005; $94: 181-5$

25. Wernovsky G, Wypij D, Jonas RA, Mayer JE Jr, Hanley FL, Hickey PR, et al. Postoperative course and hemodynamic profile after the arterial switch operation in neonates and infants. A comparison of low-flow cardiopulmonary bypass and circulatory arrest. Circulation. 1995;92:2226-35.

26. Davies G, Reid L. Growth of the alveoli and pulmonary arteries in childhood. Thorax. 1970;25:669-81.

27. Wernovsky G, Gruber P. Common congenital heart disease: presentation, management, and outcomes. In: Taeusch W, Ballard R, Gleason C, eds. Avery's diseases of the newborn. 8th ed. Philadelphia: Elsevier Saunders; 2005:p. 835.

28. Kloth RL, Baum VC. Very early extubation in children after cardiac surgery. Crit Care Med. 2002;30:787-91.

29. Allaham AH, Estrera AL, Miller CC 3rd, Achouh P, Safi HJ. Chylothorax complicating repairs of the descending and thoracoabdominal aorta. Chest. 2006; 130:1138-42.

30. Chang AC, Hanley FL, Wernovsky G, Rosenfeld HM, Wessel DL, Jonas RA, et al. Early bidirectional cavopulmonary shunt in young infants. Postoperative course and early results. Circulation. 1993;88(suppl). II149-58. 


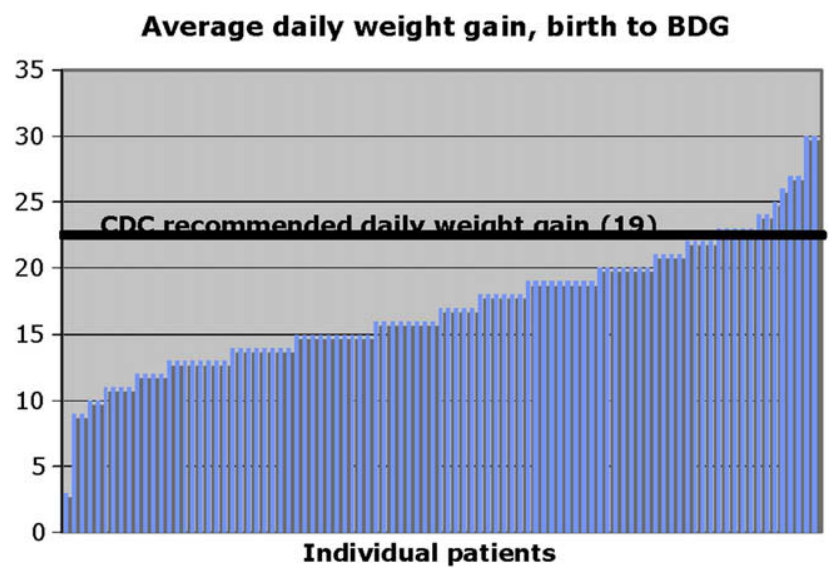

FIGURE E1. Daily weight gain (in grams per day) for individual patients between birth and BDG is shown. The dotted line indicates the recommended weight gain per day according to US Centers for Disease Control and Prevention $(C D C)$ growth curves. $B D G$, Bidirectional Glenn procedure.
Neonatal discharge

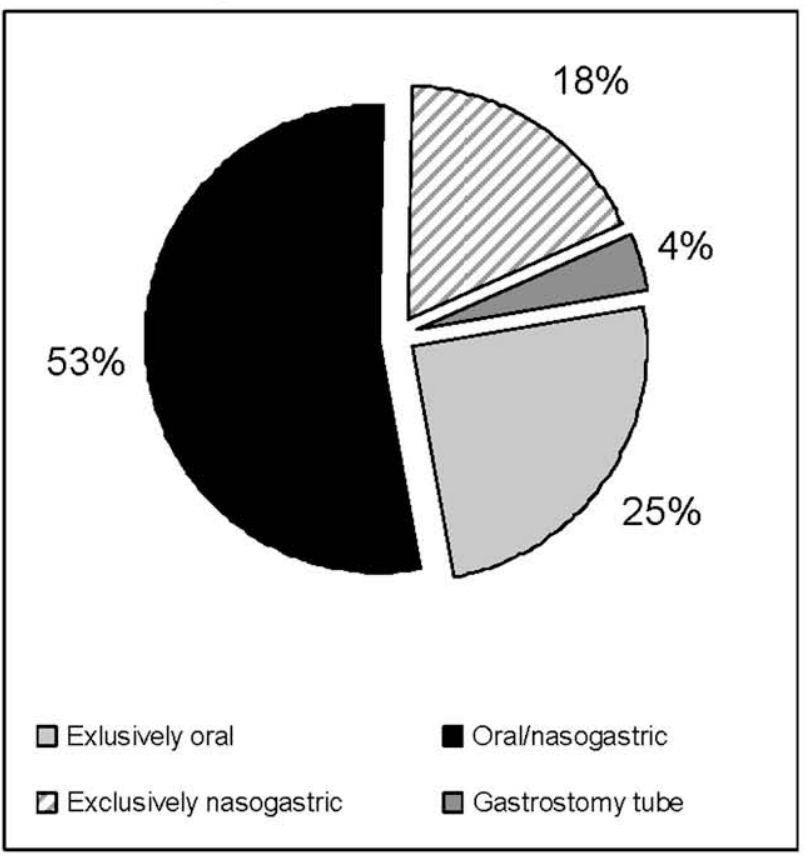

\section{BDG}

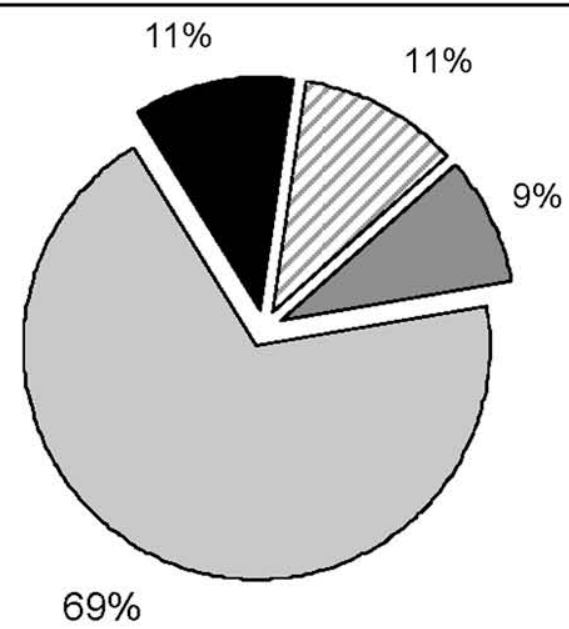

$\square$ Exlusively oral

Oral/nasogastric

$\square$ Exclusively nasogastric

Gastrostomy tube

FIGURE E2. Feeding modality at neonatal discharge versus feeding modality at the time of the bidirectional Glenn $(B D G)$ procedure. 